\title{
The chemical nature of phenolic compounds determines their toxicity and induces distinct physiological responses in Saccharomyces cerevisiae in lignocellulose hydrolysates
}

\author{
Peter Temitope Adeboye, Maurizio Bettiga and Lisbeth Olsson ${ }^{*}$
}

\begin{abstract}
We investigated the severity of the inhibitory effects of 13 phenolic compounds usually found in spruce hydrolysates (4-hydroxy-3-methoxycinnamaldehyde, homovanilyl alcohol, vanillin, syringic acid, vanillic acid, gallic acid, dihydroferulic acid, $p$-coumaric acid, hydroquinone, ferulic acid, homovanillic acid, 4-hydroxybenzoic acid and vanillylidenacetone). The effects of the selected compounds on cell growth, biomass yield and ethanol yield were studied and the toxic concentration threshold was defined for each compound. Using Ethanol Red, the popular industrial strain of Saccharomyces cerevisiae, we found the most toxic compound to be 4-hydroxy-3-methoxycinnamaldehyde which inhibited growth at a concentration of $1.8 \mathrm{mM}$. We also observed that toxicity did not generally follow a trend based on the aldehyde, acid, ketone or alcohol classification of phenolic compounds, but rather that other structural properties such as additional functional groups attached to the compound may determine its toxicity. Three distinctive growth patterns that effectively clustered all the compounds involved in the screening into three categories. We suggest that the compounds have different cellular targets, and that. We suggest that the compounds have different cellular targets and inhibitory mechanisms in the cells, also compounds who share similar pattern on cell growth may have similar inhibitory effect and mechanisms of inhibition.
\end{abstract}

Keywords: Phenolics; Toxicity; Inhibition; Tolerance; Conversion; Saccharomyces cerevisiae

\section{Introduction}

Lignocellulose, primarily made up of carbohydrates and lignin, has been billed as the most abundant material on earth (Chandel et al. 2011). Next to carbohydrates, aromatic compounds are the second most abundant class of organic compounds in nature (Boll et al. 2002). It has been claimed that aromatic compounds, including phenolics make up about $25 \%$ of the earth's biomass (Gibson and Harwood 2002). This abundance is significant to the usage of plants and plant residues as important resources in second generation biofuel and chemicals production.

Phenolic compounds are secondary metabolites that are synthesized by plants via the pentose phosphate, shikimate and phenylpropanoid pathways (Randhir et al. 2004).

\footnotetext{
* Correspondence: Lisbeth.olsson@chalmers.se

Industrial Biotechnology, Department of Chemical and Biological

Engineering, Chalmers University of Technology, Gothenburg SE-412 96,
} Sweden
They form the building blocks of lignin and they play crucial role in plants resistance to diseases and infections (Hutzler et al. 1998, Nicholson and Hammerschmidt 1992, Vance et al. 1980, Vanholme et al. 2010). Lignin in itself is a natural polymer that is primarily made up of phenylpropane units derived from guaiacol, $\mathrm{p}$-hydroxyphenol and syringol, all interconnected in a C-C bond (Dorrestijn et al. 2000, Mcdonough 1983, Nenkova et al. 2011). Phenolic compounds are directly involved in various plant physiological processes and plant defense mechanisms against microbial infections (Bhattacharya et al. 2010, Blum et al. 1999, Bravo 1998, Hutzler et al. 1998, Muller et al. 1964). In addition, their antimicrobial, antioxidant activity, and their various other dietary and pharmaceutical properties make them highly relevant to food and pharmaceutical industries (Balasundram et al. 2006, Benavente-Garcia et al. 1997, Hertog et al. 1993, Puupponen-Pimia et al. 2001, Scalbert and Mazur 2002). 
On the other hand, the beneficial effect of the antimicrobial activities of phenolic compounds which is beneficial to plants present a significant challenge to the production of second generation bioethanol and other chemicals from plant residues and lignocellulosic materials (Klinke et al. 2004). During biofuel production, plant biomasses are first subjected to pre-treatment processes and hydrolysis in order to breakdown their structures and adapt them to forms accessible by enzymes for fermentation and bioconversion. Diverse phenolic compounds are formed as residues of lignin degradation during these wood and plant residue pre-treatment processes for hydrolysate production and wood pulping (Guss 1945, Klinke et al. 2004, Larsson et al. 2000, Larsson et al. 1999b, Taherzadeh and Karimi 2007). The composition of the different phenolic compounds formed during pre-treatment varies and depend on both the plant source and the pre-treatment method (Larsson et al. 1999b). In general, the resulting mix is usually made up of phenolic acids, phenolic aldehydes, phenolic alcohols and phenolic ketones all of which are inhibitory to cells. A typical spruce hydrolysate will often consist of the phenolic compounds listed in Table 1.

The occurrence of phenolic compounds with various functional groups like aldehydes, acids, ketone and alcoholic, and the abundance of phenolic compounds in

Table 1 Table of phenolic compounds and the concentration range commonly found in spruce hydrolysates

\begin{tabular}{|c|c|}
\hline Phenolic compounds & Amount (mg/L) \\
\hline Gallic acid & $7.1-10.2$ \\
\hline Catechine & $61-71.9$ \\
\hline Vanillic acid & $3.93-71.2$ \\
\hline Syringic acid & $42.3-42.87$ \\
\hline Ferulic acid & $42.91-45.08$ \\
\hline Picein [3-( $\beta$-d-glucosyloxy)-hydroxy-acetophenone] & $0.2-1.4$ \\
\hline Pungenin[3-( $\beta-d-g l u c o s y l o x y)-4-h y d r o x y-a c e t o p h e n o n e]$ & 0.2 \\
\hline Taxifoloin & $2-33$ \\
\hline Coniferyl aldehyde & $35-301$ \\
\hline Vanillic acid & $0.01-35$ \\
\hline Vanillin & 36 \\
\hline 4-hydroxybenzoic acid & $39-81$ \\
\hline Catechol & 2 \\
\hline Acetoguaiacone & 146 \\
\hline Trans cinnamic acid & 10 \\
\hline Syringaldehyde & 107 \\
\hline
\end{tabular}

(Almeida et al. 2007b, Deflorio et al. 2011, Delvas et al. 2011, Evensen et al. 2000, Hutzler et al. 1998, Miyafuji et al. 2003).

Also, Pungenol (3',4'-hydroxy-acetophenone), Piceol (4'-hydroxyacetophenone), Trans-resveratrol, p-Coumaric acid, Coumarins, Stilbenes, Styryl pyrones, Dihydroconiferyl alcohol, Hydroquinone, Homovanillic acid have all been found in various concentrations in spruce hydrolysates (Almeida et al. 2007b, Deflorio et al. 2011, Delvas et al. 2011, Evensen et al. 2000, Hutzler et al. 1998, Miyafuji et al. 2003). wood hydrolysates present major challenges to studying them in detail. In some studies aimed at understanding phenolic compounds, compounds having similar functional groups have been grouped together while representative compounds of each group were studied (Larsson et al. 2000), presumably under the assumption that compounds having the same functional group are similar in their inhibitory activities. It has been shown that the presence of phenolic compounds in hydrolysates may determine the fermentability of hydrolysates and directly impacts on ethanol productivity of $S$. cerevisiae (Larsson et al. 1999a, Larsson et al. 2000). The effects of many selected phenolic compounds and other inhibitors on yeast fermentative conditions have been screened, and strains of S. cerevisiae engineered for phenolic tolerance have been constructed and evaluated (Delgenes et al. 1996, Gregg and Saddler 1996, Larsson et al. 2000). It is known that certain phenolic compounds such as ferulic acid and vanillin can be assimilated and converted by $S$. cerevisiae (Clausen et al. 1994, Huang et al. 1993, Vanbeneden et al. 2008) however there are concentrations at which $S$. cerevisiae cannot survive the inhibition of such compounds, the various concentrations have not been defined for phenolic compounds.

Basing our experimental work on the hypotheses that (i) different phenolic compounds have different limits of toxicity on S. cerevisiae and (ii) mechanisms and activities of inhibition among phenolic compounds may be compound-specific, we have defined the toxicity limits of 13 different phenolic compounds selected from all classes of phenolic compounds commonly found in but not limited to spruce hydrolysates. We also studied the effects of the various phenolic compounds on the growth of $S$. cerevisiae and categorised the phenolic compounds into clusters based on their effects on growth. The influence of each cluster of phenolic compounds on metabolite yields were investigated in order to draw parallels and similarities between the phenolic compounds within each cluster and to explore whether compounds within each cluster have similar influence on the physiology of S. cerevisiae, all in order to better understand phenolic inhibition in lignocellulosic fermentation.

\section{Materials and methods Yeast strain}

The industrial yeast strain S. cerevisiae Ethanol Red was used for this study. The yeast strain was obtained from the local wine-making and brewery shop in Gothenburg, Sweden.

\section{Reagents}

4-hydroxy-3-methoxycinnamaldehyde, homovanilyl alcohol, vanillin, syringic acid, vanillic acid, gallic acid, dihydroferulic acid, $p$-coumaric acid, hydroquinone, ferulic acid, 
homovanillic acid, 4-hydroxybenzoic acid and vanillylidenacetone and other reagents used for growth media preparation in the studies were procured from Sigma-Aldrich.

\section{Preparation of culture media}

The medium used for all the cultivations was Yeast Minimal Mineral Medium (YMMM) (Verduyn et al. 1992). YMMM containing single phenolic substrates was prepared for each phenolic compound using the concentrations reported under "Results".

\section{High throughput toxicity screening of phenolic compounds on S. cerevisiae}

To define the range of values within which the toxicity limits of the compounds lie, high throughput toxicity screenings were done using Bioscreen C MBR (Oy Growth Curves Ab Ltd, Finland). Several concentrations of each phenolic compound were tested. Five replicates of each concentration step were run in parallel in the following conditions: $\mathrm{T}=30^{\circ} \mathrm{C} \pm 0.1 ;$ time $=96$ hours; shaking speed setting = "maximum" optical density (OD) reading period $=15$ min; wavelength filter $=$ wideband $450-580 \mathrm{~nm}$; initial OD $=0.1$

To facilitate data comparison, the readings obtained from the bioscreen were calculated back to standard spectrophotometric measurements at $600 \mathrm{~nm}$ via the formula:

$$
O D_{\text {spectro }}=\frac{O D_{\text {Bioscreen }}}{\text { Path Length }(\mathrm{cm}) \times 1.32}
$$

Where

$\mathrm{OD}_{\text {spectro }}=$ equivalent $\mathrm{OD}$ on spectrophotometer at $600 \mathrm{~nm}$ $\mathrm{OD}_{\text {Bioscreen }}=$ measured $\mathrm{OD}$ on the bioscreen

$$
\text { PathLength }=\frac{\text { volume }(m l)}{r^{2} X \pi}
$$

Where: volume $=$ culture volume in a well in the bioscreen plate; $r$ = radius of the well.

Non-linearity at higher cell densities was corrected as described by Warringer et al., (Warringer and Blomberg 2003) using the formula:

$$
\begin{aligned}
\text { ODcor }= & \text { ODobs }+\left(O D^{2} \text { obs } * 0.449\right) \\
& +\left(O D^{3} \text { obs } * 0.191\right)
\end{aligned}
$$

Where: ODcor $=$ the corrected OD and ODobs = the observed OD values, from which the average blank has been subtracted.

Aerobic batch cultivations were carried out in $100 \mathrm{ml}$ or $250 \mathrm{ml}$ baffled Erlenmeyer flasks (SIMAX, Czech Republic), containing $20 \mathrm{ml}$ and $50 \mathrm{ml}$ medium, respectively.

\section{OD measurement of culture}

Growth measurement for shake flask cultivations was done by measuring the turbidity of the culture at $\mathrm{A}_{600 \mathrm{~nm}}$ using a Thermo Scientific GENESYS 20 Visible Spectrophotometer.

\section{Determination of dry cell weight}

Determination of Dry Cell Weight (DCW) was done in duplicates. Cells were harvested by filtration using predried and weighed filter paper discs of $0.45 \mu \mathrm{m}$ pore size (Sartorius Stedim Biotech, Goettingen, Germany) on a water tap vacuum filter unit (Sartorius Stedim Biotech, Goettingen, Germany). The filter paper discs were dried in a microwave at $120 \mathrm{~W}$ for 15 minutes, weighed again and the biomass concentrated was calculated from the difference. DCW data were used for the calculation of biomass yield.

\section{Analysis of metabolites}

Analysis of ethanol, glycerol and acetate from the cultivation was performed by high performance liquid chromatography (HPLC) using a Dionex Ultimate 3000 HPLC unit (Thermo scientific, Dionex Corporation, Sunnyvale, USA) equipped with an Aminex HPX-87H (Biorad, USA) column of length $300 \mathrm{~mm}$ and diameter $7.8 \mathrm{~mm}$, which was packed with $9 \mu \mathrm{m}$ particles. A column temperature of $45^{\circ} \mathrm{C}$ was used for analysis and $5 \mathrm{mM} \mathrm{H} 2 \mathrm{SO} 4$ was used as the mobile phase with a flow rate of $0.6 \mathrm{ml} / \mathrm{min}$ throughout the analysis. A Shodex RI-101 RI detector and an Ultimate 3000 VWD 3100 variable wavelength ultraviolet detector coupled to the HPLC unit were used to quantify the metabolites.

\section{Determination yields}

Yields (Yi/s) of ethanol, glycerol, acetate and biomass from the total consumed substrate (glucose) were calculated during the exponential growth phase by plotting each of the products against the total consumed glucose. The yield for each product was then obtained as the slope of the plot. Average values of biological replicates were used as the final yield for each culture condition.

\section{Establishing concentration ladder of compounds}

A concentration series was set up in increasing order for each compound to be screened for effect on S. cerevisiae. Since toxicity varies widely and using a universal concentration series for all of the compounds was not feasible, we determined consistent ratios of increase between consecutive points in the concentration series for all compounds to allow comparison of toxicity among the various compounds.

\section{Determination of toxicity limits}

The toxicity limits of the different phenolic compounds were determined based on the aspect (maximum specific 
growth rates or final OD or elongation of lag phase) at which the yeast cultivations were most affected. The maximum specific growth rate of the $S$. cerevisiae in the presence of increasing concentrations of phenolic compounds were determined with increasing concentration of the compounds until cell growth stopped.

\section{Statistical validation of data}

All experimental data were subjected to Student t-test to determine the significance level with respect to the control. The number of replicates varied from 3 to 7 , depending on the experiment. Therefore, t-tests for twosamples assuming unequal variances were performed with a significance level of probability set at $\mathrm{p}<0.05$. All error bars were standard deviations of multiple measurements of each parameter, all derived from biological replicates.

\section{Results}

\section{Effect of compounds on S. cerevisiae growth pattern}

We hypothesized that the physiological effect of each phenolic compound on $S$. cerevisiae would be unique and have phenotypic traits demonstrated in the growth pattern of $S$. cerevisiae. Yeast was grown in the presence of 4-hydroxy-3-methoxycinnamaldehyde, homovanilyl alcohol, p-coumaric acid, hydroquinone, ferulic acid, vanillin, syringic acid, homovanillic acid, 4-hydroxybenzoic acid, vanillic acid, gallic acid, vanillylidenacetone and dihydroferulic acid and the impact of the compounds on growth profiles, maximum specific growth rates and culture turbidity was assessed. S. cerevisiae had three unique growth patterns that distinctively grouped all the phenolic compounds into three clusters which we named cluster 1 , cluster 2 and cluster 3 (Figure 1).

The culture containing phenolic compounds in cluster 1, exhibited lag phase elongation which increased with increasing concentration of phenolic compounds in the medium until a concentration of compound is attained at which growth was no longer possible. The second and third growth pattern clusters showed no elongation of lag phase. The phenolic compounds also had specific effects on maximum specific growth rate within their clusters. In cultures containing cluster 1 compounds, maximum specific growth rate decreased with increasing concentration of phenolic compound until a concentration is attained at which growth finally stopped (Additional file 1: Figure S1). Similarly, in cultures containing the cluster 2 compounds, the maximum specific growth rate reduced with increasing concentration of phenolic compound until a concentration is attained at which growth was no longer possible

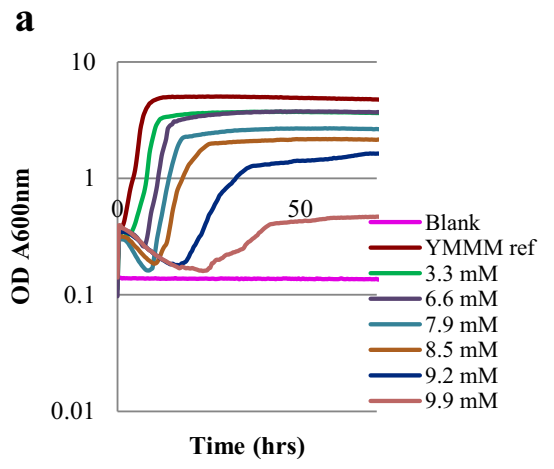

c

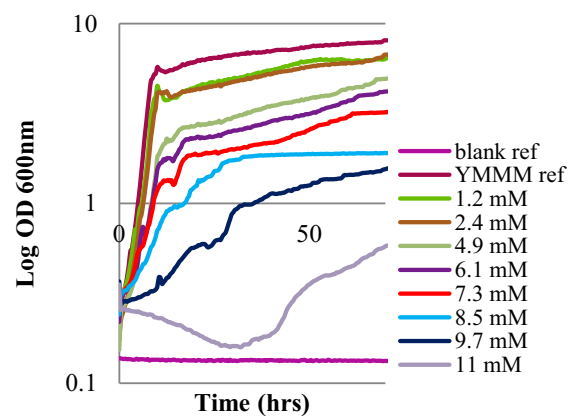

b

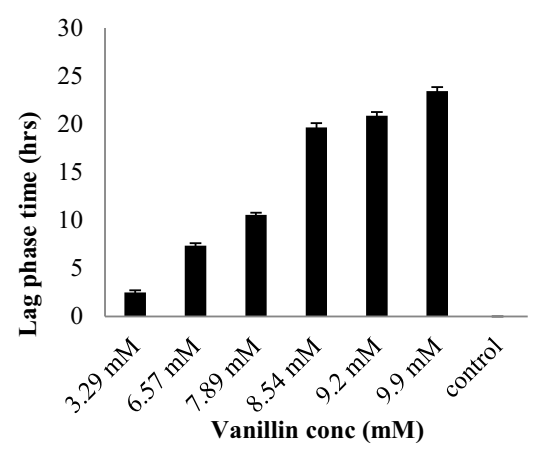

d

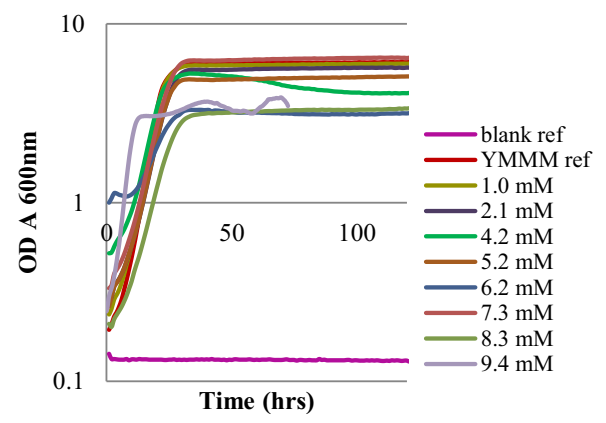

Figure 1 Three distinct growth profiles of Saccharomyces cerevisiae in the presence of phenolic compounds with: a. vanillin; c. $p$-Coumaric acid; $\mathbf{d}$. Vanillylidenacetone representing common growth profiles within the three cluster groups. Elongation in lag phase was only shown in figure $\mathbf{b}$ for the first cluster since there was no lag phase elongation in the second and third clusters of compounds. 
(Additional file 1: Figure S1b). In cultures contaning the third cluster of compounds however (as illustrated in Additional file 1: Figure S1c), the maximum specific growth rate remained constant until the concentration was attained at which growth was no longer possible.

The determination of biomass formation in the cultivations was limited to OD measurements on the bioscreen. In cultivations containing cluster 1 compounds, the OD of the yeast cultivations decreased with increasing concentration of phenolic compound until a concentration is attained at which growth stopped. As illustrated in Additional file 2: Figures S2a and S2, the reduction in OD was observed to be valid for the first and second clusters of compounds. In the third cluster however, although a reduction in the final OD was observed (Additional file 2: Figure S2c), the observed reduction was not as strong as in the first two compound clusters.

This categorization growth profile groups the thirteen phenolic compounds as;

- Cluster1: 4-hydroxy-3-methoxycinnamaldehyde, Homovanilyl alcohol, Vanillin, Syringic acid and Dihydroferulic acid.

- Cluster 2: p-Coumaric acid, hydroquinone, Ferulic acid, Homovanillic acid and 4-hydroxybenzoic acid and

- Cluster 3: Vanillic acid, Gallic acid and Vanillylidenacetone.

\section{Different phenolic compounds have different limits of toxicity}

In experimentally defining the concentration threshold at which the selected phenolic compounds completely inhibit yeast growth, we conducted toxicity screening on the phenolic compounds. During the screening, the maximum specific growth rates of the cultures when growth was last observed ranged between $0.07 \mathrm{~h}^{-1}$ and $0.09 \mathrm{~h}^{-1}$, this was about $20 \%$ of the maximum specific growth rate of the control. In the presence of another set of phenolic compounds in which the cells experienced increased lag phase and reduced biomass with increasing concentration of the phenolic compounds, the concentration at which the cells last showed observable growth had an elongated the lag phase of about 5 times that of the control, the cells stopped growing in higher concentrations. In the third category, the cells suddenly stopped growing after a certain concentration and this concentration was noted. The toxicity screening revealed a wide range of toxicity among the compounds (Figure 2). The screening also revealed that each compound has a toxicity limit that is not necessarily based on its classification as an acid, alcohol, aldehyde or a ketone. Coniferyl aldehyde had the highest toxicity, becoming extremely toxic at $1.4 \mathrm{mM}$ for cells to grow while syringic acid is the least toxic with cell growth

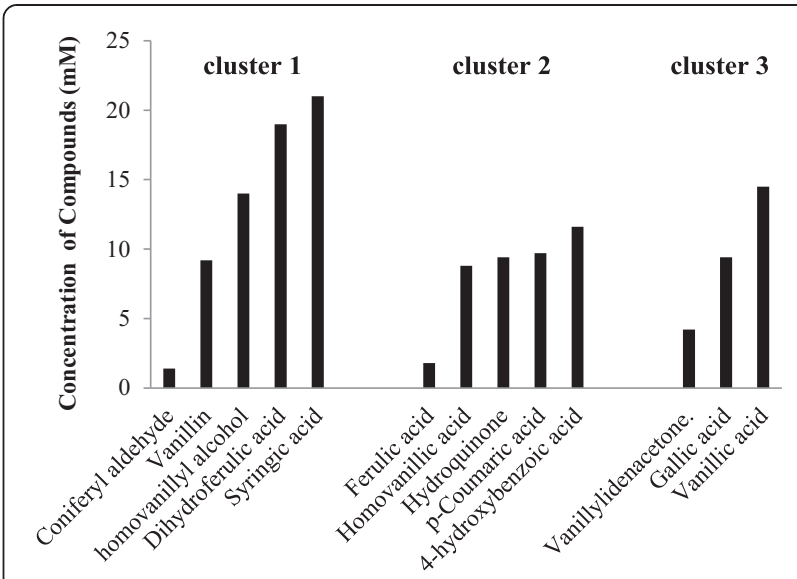

Figure 2 Bar chart showing concentrations at which different phenolic compounds within each growth-based cluster became too toxic for the growth of Saccharomyces cerevisiae.

continuing to be recorded at $22 \mathrm{mM}$. Further concentration increase in syringic acid was limited by strong interference in measurements as a result of the deep colouration of the medium (Figure 2).

\section{Effects of toxic concentrations of phenolic compounds on ethanol and biomass titres and yields}

In the next step we investigated whether compounds clustered together by order of growth pattern would also have similar effect on the yeast cell physiology. A pair of compounds was selected from each cluster and their effects on ethanol, acetate, glycerol and biomass yields were determined. Syringic and dihydroferulic acids were selected from the first cluster, homovanillic and 4hydroxybenzoic acids were selected from the second cluster and vanillylidenacetone and gallic acid were selected from the third cluster. The compounds were added to the cultivation medium at their respective toxicity limit concentrations of $18.0 \mathrm{mM}$ syringic acid, $18.0 \mathrm{mM}$ dihydroferulic acid, $9.0 \mathrm{mM}$ homovanillic acid, $11.0 \mathrm{mM} 4$ hydroxybenzoic acid, $4.2 \mathrm{mM}$ vanillylidenacetone and $9.4 \mathrm{mM}$ gallic acid.

Glucose consumption was particularly delayed in dihydroferulic acid cultivations (Figure 3). No significant difference in glucose consumption was observed between any of the cultures containing syringic, homovanillic, 4hydroxybenzoic, gallic acid or vanillylidenacetone, and the control (Figure 3). Ethanol assimilation after glucose depletion during the respiratory growth phase was slowed down for all cultures with the phenolic compounds except for cultures containing syringic acid.

Further comparison within each cluster was done based on the yields of ethanol, glycerol, acetate and biomass. Ethanol yield in dihydroferulic acid and syringic acid cultures were similar at $0.43 \pm 0.01(\mathrm{~g} / \mathrm{g})$ and $0.38 \pm 0.03(\mathrm{~g} / \mathrm{g})$ respectively (Figure 4). The yield of glycerol in dihydroferulic 


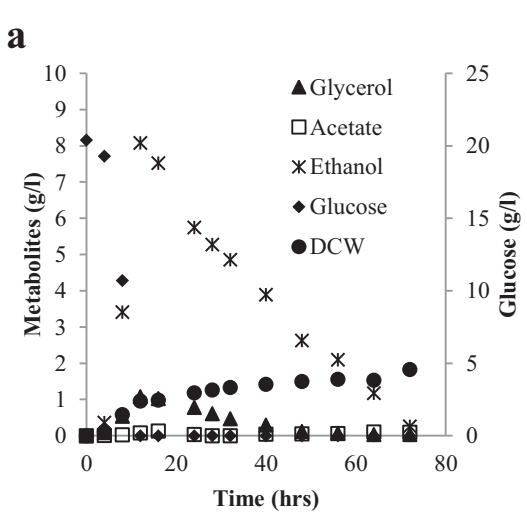

c
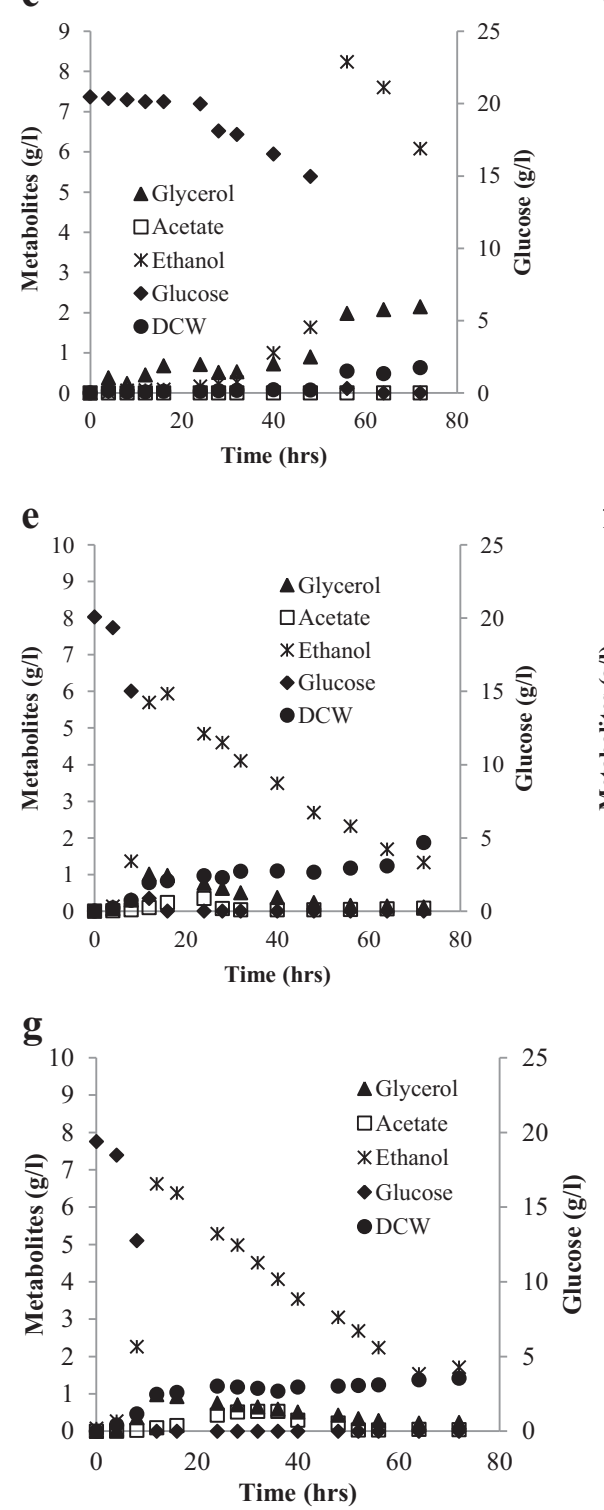

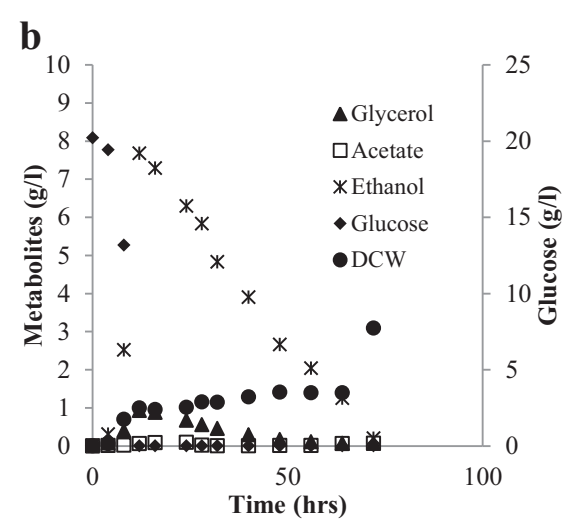

d
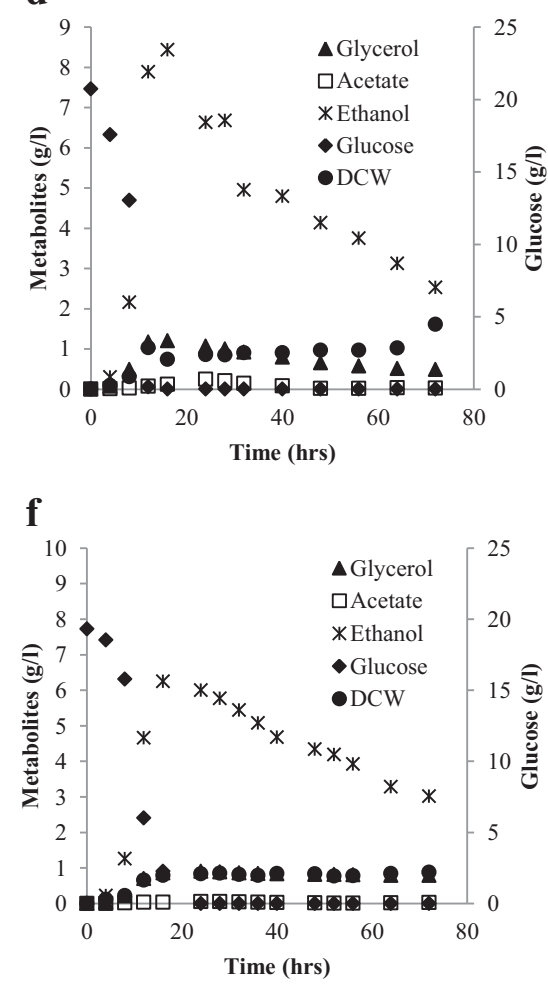

Figure 3 Metabolite profiles representatives of Saccharomyces cerevisiae cultivations in the presence of: a. YMMM; b. syringic acid; c. dihydroferulic acid; d. homovanillic acid; e. 4-hydroxybenzoic acid; f. vanillylidenacetone and g. gallic acid. 


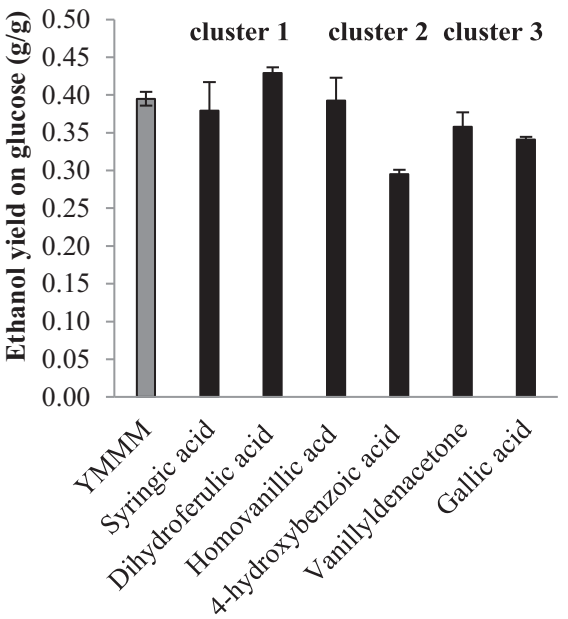

c

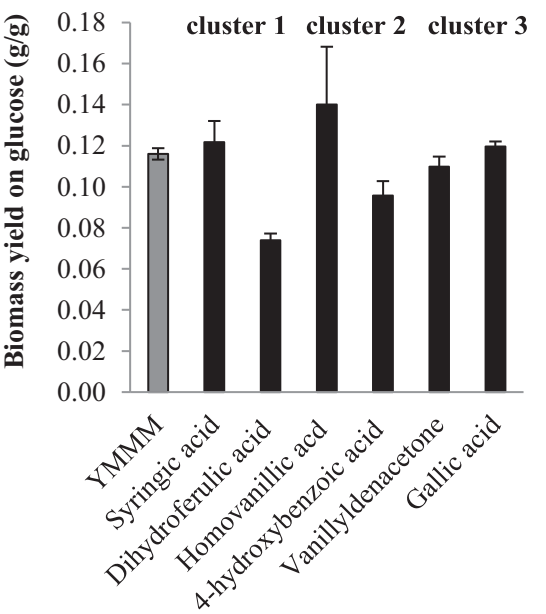

b

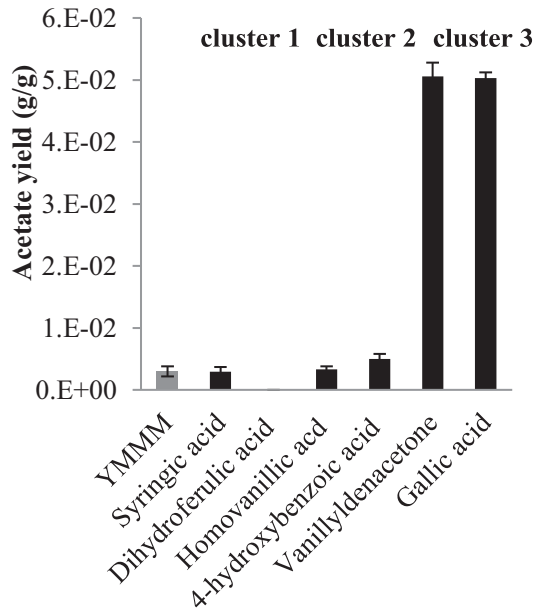

d

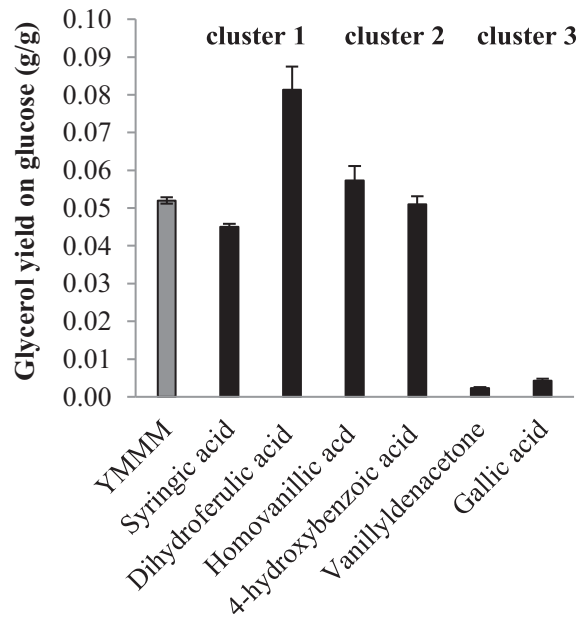

Figure 4 Intra-cluster comparison of the effects of phenolic compounds on: a. ethanol yield; b. acetate yield; c. biomass yield; and d. glycerol yield from glucose. Syringic and dihydroferulic acids were selected from cluster 1, homovanillic and 4-hydroxybenzoic acid from cluster 2, vanillylidenacetone and gallic acid from cluster 3.

acid containing cultures was higher than in syringic acid containing cultures with yields of $0.081 \pm 0.006(\mathrm{~g} / \mathrm{g})$ and $0.045 \pm 0.001(\mathrm{~g} / \mathrm{g})$ respectively. The most outstanding difference between this pair however was that acetate was not found in dihydroferulic acid cultivations while acetate yield was $0.003(\mathrm{~g} / \mathrm{g})$ in syringic acid cultures which was the same as that of the control (Figure 4).

Ethanol, acetate and biomass yields in homovanillic acid cultures were significantly different to 4-hydroxybenzoic acid cultures. Ethanol yield at $0.39 \pm 0.03(\mathrm{~g} / \mathrm{g})$ and biomass at $0.14 \pm 0.03(\mathrm{~g} / \mathrm{g})$ were higher in homovanillic acid containing cultures compared with $0.3 \pm 0.01(\mathrm{~g} / \mathrm{g})$ and $0.096 \pm 0.007(\mathrm{~g} / \mathrm{g})$ respectively for ethanol and biomass yields in 4-hydroxybenzoic acid. Acetate yield was lower in homovanillic acid cultures at $0.003 \pm 0.0005$ (g/g) compared to $0.005 \pm 0.001(\mathrm{~g} / \mathrm{g})$ in 4-hydroxybenzoic acid. However, glycerol yields of homovanillic and 4hydroxybenzoic acids were similar at $0.057 \pm 0.004(\mathrm{~g} / \mathrm{g})$ and $0.051 \pm 0.002(\mathrm{~g} / \mathrm{g})$ respectively. Results for vanillylidenacetone and gallic acid (cluster 3 ) proved very consistent for ethanol, biomass, acetate and glycerol yields (Figure 4). A significant difference was observed between glycerol and acetate yields in the third cluster (vanillylidenacetone and gallic acid) and those in the other two clusters and the control. Glycerol yield in cluster 3 was 10 times lower at $0.002 \pm 0.0002(\mathrm{~g} / \mathrm{g})$ for vanillylidenacetone and $0.004 \pm 0.0006(\mathrm{~g} / \mathrm{g})$ for gallic acid cultures, and acetate yield was higher by 10 times at $0.051 \pm 0.002(\mathrm{~g} / \mathrm{g})$ for vanillylidenacetone and $0.05 \pm 0.001(\mathrm{~g} / \mathrm{g})$ for gallic acid than in both YMMM and the other two clusters (Figure 4). 
Overall ethanol yield in dihydroferulic acid was the highest at 0.43 (g/g) while 4-hydroxybenzoic acid had the lowest ethanol yield and the highest acetate yields of all cultures. The similarities in effect of each clustered pair of phenolic compounds on yeast metabolism indicate that compounds in the same cluster have similar inhibitory effects on yeast.

\section{Discussion}

In this study, we classified 13 different phenolic compounds commonly found in lignocellulosic hydrolysates according to their effect on S. cerevisiae growth. In particular, we showed that (i) the concentration that induces inhibitory effects is highly variable among phenolic compounds and it does not follow the order of phenolic aldehydes and ketones of being the most toxic, followed by acids and alcohols, respectively (Almeida et al. 2007a, Klinke et al. 2003) (ii) the influence of phenolic compounds on $S$. cerevisiae growth follows three major patterns; (iii) different compounds have distinct effect not only on biomass formation but also on the production of ethanol, acetate and glycerol.

Phenolic compounds have often been grouped and ordered as aldehydes, phenolic ketones, phenolic acids and phenolic alcohols, and their potency as inhibitors has largely been believed to reflect the same order. Phenolic aldehydes have generally been regarded as the most inhibitory while phenolic acids and alcohols tend to be seen as the least toxic (Almeida et al. 2007a, Klinke et al. 2003). In this study however, we demonstrated that the toxicity of phenolic compounds does not follow the assumed order in the subset of compounds we selected and is not dependent only on the recognised aldehyde, carboxylic acid, alcohol and ketone functional groups. Based on our results, we speculate that the inhibitory effects of phenolic compounds is a function of the combination of the occurrence of functional side groups (such as the methoxy and hydroxyl groups) and occurrence of unsaturated bonds in the structure of the compounds regardless of the categorization of the compounds as aldehydes, acid, alcohols or ketones. An example that supports this speculation is the different toxicities of coniferyl aldehyde (1.1 mM), ferulic acid (1.8 mM), and vanillin $(9.7 \mathrm{mM})$ see Figure 5 . Our results thus show that although coniferyl aldehyde is the most toxic at $1.1 \mathrm{mM}$, ferulic acid is more toxic at a toxicity limit of $1.8 \mathrm{mM}$ than vanillin which is an aldehyde with a toxicity limit of $9.2 \mathrm{mM}$. The major difference between vanillin and coniferyl aldehyde is the occurrence of 2 extra carbon atoms sharing a double bond and linking the aldehyde group to the aromatic ring in coniferyl aldehyde. Ferulic acid also possesses 2 extra carbon atoms sharing a double bond and linking the carboxylic acid group to the aromatic ring. We speculate that these chemical features significantly

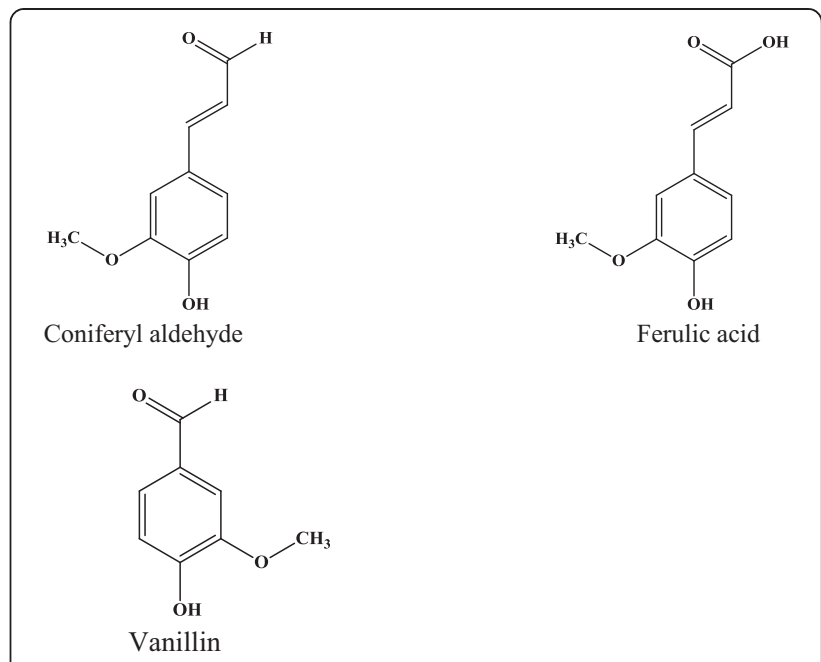

Figure 5 Structures of coniferyl aldehyde, ferulic acid and vanillin.

contribute to the toxicity of coniferyl aldehyde and ferulic acid, in line with earlier findings that the occurrence and positions of functional side groups as well as the presence of unsaturated carbon to carbon bonds influence the biological reactions and inhibitory activities of phenolic compounds in bacteria as well as their antioxidant activity in human (Ramaswam et al. 1972, Rice-Evans et al. 1996).

Three distinct growth patterns among the thirteen different phenolic compounds screened was observed, suggesting that compounds belonging to the same cluster display similarity in mechanisms of inhibition mechanisms. The similarity of ethanol yields between compounds representing cluster 1, acetate and glycerol yields in cluster 2 and the strong correlation of effects of vanillylidenacetone and Gallic acid (cluster 3) on ethanol, glycerol, acetate and biomass yields suggest that compounds belonging to the same cluster have similar inhibitory activity on yeast.

Phenolic compounds have been shown to reduce yields of ethanol and alter glycerol and acetate yields from $S$. cerevisiae fermentations. Studies by Ando et al., (1986), revealed that syringaldehyde, $m$-hydroxybenzoic acid and vanillic acid did not inhibit ethanolic fermentation while coniferyl aldehyde led to poor fermentation and drastically reduced ethanol yield. Larsson et al. (2000) also corroborated the severely inhibitory effect of coniferyl aldehyde in their study. Our results aligned with previous studies which strengthens our confidence in the toxicity ranking of all our tested phenolic compounds in which we found coniferyl aldehyde to be the most toxic phenolic compound. The influence of the phenolic compounds on yeast physiology was mostly visible through their impacts on glycerol, biomass and acetate yields among the compound clusters. Absence of quantifiable acetate production, poor growth and delayed glucose utilisation characterized 
the dihydroferulic acid cultivations. Known conditions that may prevent the accumulation of acetate in cultures include, disruption of acetaldehyde dehydrogenases, low formation of acetaldehyde coupled with effective oxidation of acetate by acetyl-coA-synthetase or the presence of low amount of glucose in cultures such that respirofermentative growth cannot take place (Postma et al. 1989). The presence of ethanol $(0.43 \mathrm{~g} / \mathrm{g})$ and glycerol did confirm a respirofermentative growth for the yeast under these cultivation conditions. Speculatively, the apparent absence of acetate in the cultivation may resemble a situation where low activity of Cytosolic $\mathrm{Mg}^{2+}$ and Mitochondrial $\mathrm{K}^{+}$acetaldehyde dehydrogenases Ald6p and Ald4p is present. Mutants of $A L D 6$ have been shown to substantially reduce acetate production while significantly increasing glycerol production. Double mutants of Ald6p and Ald4p have been shown to have delayed growth, and delayed glucose consumption (Remize et al. 2000), as observed in our dihydroferulic acid cultivation. It is therefore tempting to speculate that these two enzymes might be a direct or indirect target of ferulic acid, although this goes beyond the purpose of this article and deserves further investigation. Glycerol being a metabolite strongly associated with different types of stress in cells, the particularly high glycerol titre and yield in the cultivation of dihydroferulic acid is indicative of the cells being under significant stress from dihydroferulic acid although we have not defined the type of stress imposed at this stage of the study.

Although glucose consumption was delayed in dihydroferulic acid cultivations, ethanol yield was high and slightly superior to the ethanol yield in the control. Ethanol yields recorded in this study were high, ranging from $0.3 \pm 0.01(\mathrm{~g} / \mathrm{g})$ in 4-hydroxybenzoic acid cultures to $0.43(\mathrm{~g} / \mathrm{g})$ in dihydroferulic acid cultures, we attribute this to the ability of the cells to adapt to the compounds and in certain cases convert some of them such as 4-hydroxybenzoic acid and dihydroferulic acid and eventually recover, thus bringing to attention and supporting findings of the natural ability of Saccharomyces cerevisiae to tolerate phenolic compounds (Stratford et al. 2007).

In conclusion, different phenolic compounds often present in lignocellulosic hydrolysates have toxicity limits that are not necessarily similar even between phenolic compounds sharing the same functional groups. An example of this would be the significant difference between ferulic acid and $p$-coumaric acid which we discovered in this study to respectively possess toxicity limits of $1.8 \mathrm{mM}$ and $9.7 \mathrm{mM}$. The experiments showed that phenolics rich substrates may be fermentable since fermentability depends on the concentration and the nature of phenolic compounds present in them. Indications also emerged from the present study that mechanisms of inhibition among phenolic compounds are dissimilar and may not be defined by the classes of phenolic compounds (aldehydes, acids, alcohols and ketones) as they are currently known. Further studies involving investigation of gene regulation and varying enzymatic studies are needed to draw conclusions on the specificity of phenolic compounds inhibition in Saccharomyces cerevisiae.

\section{Additional files}

Additional file 1: Figure S1. Effects of increasing concentration of phenolic compounds on maximum specific growth rates with: a. vanillin; b. $p$-coumaric acid; c. vanillylidenacetone representing the effects of phenolic compounds in clusters 1, 2, and 3 respectively, created according to the observed growth profile.

Additional file 2: Figure S2. Effects of increasing concentration of phenolic compounds on final optical densities of cultivations with: a. vanillin; b. p-coumaric acid; c. vanillylidenacetone representing the effects $\mathrm{f}$ phenolic compounds in clusters 1,2, and 3 respectively, created according to the observed growth profile.

\section{Competing interests}

The authors of this work declare that they have no competing interests.

\section{Authors' contributions}

Experimental design, work and writing of the manuscript were carried out by PTA. Experimental design and the subsequent manuscript were reviewed by MA. and LO. All authors read and approved the final manuscript.

\section{Acknowledgements}

This work was financially supported with a grant from the Swedish Research Council, Vetenskapsrådet.

Received: 2 March 2014 Accepted: 4 May 2014

Published online: 29 May 2014

\section{References}

Ando S, Arai I, Kiyoto K, Hanai S (1986) Identification of Aromatic Monomers in Steam-Exploded Poplar and Their Influences on Ethanol Fermentation by Saccharomyces cerevisiae. Journal of Fermentation Technology 64(6):567-570

Almeida J, Modig T, Petersson A, Hähn-Hägerdal B, Liden G, Gorwa-Grauslund M (2007a) Increased tolerance and conversion of inhibitors in lignocellulosic hydrolysates by Saccharomyces cerevisiae. J Chem Technol Biotechnol 82:340-349

Balasundram N, Sundram K, Samman S (2006) Phenolic compounds in plants and agri-industrial by-products: antioxidant activity, occurrence, and potential uses. Food Chem 99:191-203

Benavente-Garcia O, Castillo J, Marin FR, Ortuno A, Del Rio JA (1997) Uses and properties of citrus flavonoids. J Agric Food Chem 45:4505-4515

Bhattacharya A, Sood P, Citovsky V (2010) The roles of plant phenolics in defence and communication during Agrobacterium and Rhizobium infection. Mol Plant Pathol 11:705-719

Blum U, Shafer SR, Lehman ME (1999) Evidence for inhibitory allelopathic interactions involving phenolic acids in field soils: concepts vs. an experimental model. Crit Rev Plant Sci 18:673-693

Boll M, Fuchs G, Heider J (2002) Anaerobic oxidation of aromatic compounds and hydrocarbons. Curr Opin Chem Biol 6:604-611

Bravo L (1998) Polyphenols: chemistry, dietary sources, metabolism, and nutritional significance. Nutr Rev 56:317-333

Chandel AK, da Silva SS, Singh OV (2011) Detoxification of lignocellulosic hydrolysates for improved bioethanol production. Biofuel Production - Recent Developments and Prospects, Dr. Marco Aurelio Dos Santos Bernardes (Ed.), ISBN: 978-953-307-478-8, InTech, DOI: 10.5772/16454

Clausen M, Lamb CJ, Megnet R, Doerner PW (1994) Pad1 encodes phenylacrylic acid decarboxylase which confers resistance to cinnamic acid in Saccharomyces cerevisiae. Gene 142:107-112

Deflorio G, Horgan G, Woodward S, Fossdal CG (2011) Gene expression profiles, phenolics and lignin of Sitka spruce bark and sapwood before and after wounding and inoculation with Heterobasidion annosum. Physiol Mol Plant Pathol 75:180-187 
Delgenes JP, Moletta R, Navarro JM (1996) Effects of lignocellulose degradation products on ethanol fermentations of glucose and xylose by Saccharomyces cerevisiae, Zymomonas mobilis, Pichia stipitis, and Candida shehatae. Enzym Microb Technol 19:220-225

Delvas N, Bauce E, Labbe C, Ollevier T, Belanger R (2011) Phenolic compounds that confer resistance to spruce budworm. Entomologia Experimentalis Et Applicata 141:35-44

Dorrestijn E, Laarhoven LJJ, Arends IWCE, Mulder P (2000) The occurrence and reactivity of phenoxyl linkages in lignin and low rank coal. J Anal Appl Pyrolysis 54:153-192

Evensen PC, Solheim H, Hoiland K, Stenersen J (2000) Induced resistance of Norway spruce, variation of phenolic compounds and their effects on fungal pathogens. For Pathol 30:97-108

Gibson J, Harwood SC (2002) Metabolic diversity in aromatic compound utilization by anaerobic microbes. Annu Rev Microbiol 56:345-369

Gregg D, Saddler JN (1996) A techno-economic assessment of the pretreatment and fractionation steps of a biomass-to-ethanol process. Appl Biochem Biotechnol 57-8:711-727

Guss CO (1945) Acid hydrolysis of waste wood for use in plastics United States department of agriculture forest service. Forest Products Laboratory, Madison, Wisconsin Report No, R1481

Hertog MGL, Feskens EJM, Hollman PCH, Katan MB, Kromhout D (1993) Dietary antioxidant flavonoids and risk of coronary heart-disease - the Zutphen Elderly study. Lancet 342:1007-1011

Huang ZX, Dostal L, Rosazza JPN (1993) Microbial transformations of ferulic acid by Saccharomyces cerevisiae and Pseudomonas fluorescens. Appl Environ Microbiol 59:2244-2250

Hutzler P, Fischbach R, Heller W, Jungblut TP, Reuber S, Schmitz R, Veit M, Weissenbock G, Schnitzler JP (1998) Tissue localization of phenolic compounds in plants by confocal laser scanning microscopy. J Exp Bot 49:953-965

Klinke HB, Olsson L, Thomsen AB, Ahring BK (2003) Potential inhibitors from wet oxidation of wheat straw and their effect on ethanol production of Saccharomyces cerevisiae: wet oxidation and fermentation by yeast. Biotechnol Bioeng 81:738-747

Klinke HB, Thomsen AB, Ahring BK (2004) Inhibition of ethanol-producing yeast and bacteria by degradation products produced during pre-treatment of biomass. Appl Microbiol Biotechnol 66:10-26

Larsson S, Reimann A, Nilvebrant NO, Jonsson L (1999a) Comparison of different methods for the detoxification of lignocellulose hydrolyzates of spruce. Appl Biochem Biotechnol 77-9:91-103

Larsson S, Palmqvist E, Hahn-Hagerdal B, Tengborg C, Stenberg K, Zacchi G, Nilvebrant NO (1999b) The generation of fermentation inhibitors during dilute acid hydrolysis of softwood. Enzym Microb Technol 24:151-159

Larsson S, Quintana-Sainz A, Reimann A, Nilvebrant NO, Jonsson LJ (2000) Influence of lignocellulose-derived aromatic compounds on oxygen-limited growth and ethanolic fermentation by Saccharomyces cerevisiae. Appl Biochem Biotechnol 84-6:617-632

Mcdonough TJ (1983) Wood chemistry - fundamentals and applications - Sjostrom E. J Am Chem Soc 105:4503-4503

Miyafuji H, Danner H, Neureiter M, Thomasser C, Bvochora J, Szolar O, Braun R (2003) Detoxification of wood hydrolysates with wood charcoal for increasing the fermentability of hydrolysates. Enzym Microb Technol 32:396-400

Muller CH, Haines BL, Muller WH (1964) Volatile growth inhibitors produced by Aromatic Shrubs. Science 143:471

Nenkova S, Radoykova T, Stanulov K (2011) Preparation and antioxidant properties of biomass low molecular phenolic compounds. Journal of the University of Chemical Technology and Metallurgy 46:109-120

Nicholson RL, Hammerschmidt R (1992) Phenolic-compounds and their role in disease resistance. Annu Rev Phytopathol 30:369-389

Postma E, Verduyn C, Scheffers WA, Van Dijken JP (1989) Enzymic analysis of the crabtree effect in glucose-limited chemostat cultures of Saccharomyces cerevisiae. Appl Environ Microbiol 55:468-477

Puupponen-Pimia R, Nohynek L, Meier C, Kahkonen M, Heinonen M, Hopia A, Oksman-Caldentey KM (2001) Antimicrobial properties of phenolic compounds from berries. J Appl Microbiol 90:494-507

Ramaswam AS, Rao KH, Sirsi M, Jayarama S (1972) Antibacterial action of some naturally occurring citrus bioflavonoids. Indian J Exp Biol 10:72
Randhir R, Lin YT, Shetty K (2004) Phenolics, their antioxidant and antimicrobial activity in dark germinated fenugreek sprouts in response to peptide and phytochemical elicitors. Asia Pac J Clin Nutr 13:295-307

Remize F, Andrieu E, Dequin S (2000) Engineering of the pyruvate dehydrogenase bypass in Saccharomyces cerevisiae: role of the cytosolic Mg2+ and mitochondrial $\mathrm{K}+$ acetaldehyde dehydrogenases Ald6p and Ald4p in acetate formation during alcoholic fermentation. Appl Environ Microbiol 66:3151-3159

Rice-Evans CA, Miller NJ, Paganga G (1996) Structure-antioxidant activity relationships of flavonoids and phenolic acids. Free Radic Biol Med 20:933-956

Scalbert A, Mazur A (2002) Dietary polyphenols and the prevention of cardiovascular diseases - state of the art. Cardiovascular Dieseases 2002:351-357

Stratford M, Plumridge A, Archer DB (2007) Decarboxylation of sorbic acid by spoilage yeasts is associated with the PAD1 gene. Appl Environ Microbiol 73:6534-6542

Taherzadeh MJ, Karimi K (2007) Acid-based hydrolysis processes for ethanol from lignocellulosic materials: a review. Bioresources 2:472-499

Vanbeneden N, Gils F, Delvaux F, Delvaux FR (2008) Formation of 4-vinyl and 4-ethyl derivatives from hydroxycinnamic acids: occurrence of volatile phenolic flavour compounds in beer and distribution of Pad1-activity among brewing yeasts. Food Chem 107:221-230

Vance CP, Kirk TK, Sherwood RT (1980) Lignification as a mechanism of disease resistance. Annu Rev Phytopathol 18:259-288

Vanholme R, Demedts B, Morreel K, Ralph J, Boerjan W (2010) Lignin biosynthesis and structure. Plant Physiol 153:895-905

Verduyn C, Postma E, Scheffers WA, Van Dijken JP (1992) Effect of benzoic acid on metabolic fluxes in yeasts: a continuous-culture study on the regulation of respiration and alcoholic fermentation. Yeast 8:501-517

Warringer J, Blomberg A (2003) Automated screening in environmental arrays allows analysis of quantitative phenotypic profiles in Saccharomyces cerevisiae. Yeast 20:53-67

\section{doi:10.1186/s13568-014-0046-7}

Cite this article as: Adeboye et al:: The chemical nature of phenolic compounds determines their toxicity and induces distinct physiological responses in Saccharomyces cerevisiae in lignocellulose hydrolysates. AMB Express 2014 4:46.

\section{Submit your manuscript to a SpringerOpen ${ }^{\odot}$ journal and benefit from:}

- Convenient online submission

- Rigorous peer review

- Immediate publication on acceptance

- Open access: articles freely available online

- High visibility within the field

- Retaining the copyright to your article

Submit your next manuscript at $\gg$ springeropen.com 